\title{
Robust FETI solvers for multiscale elliptic PDEs
}

\author{
Clemens Pechstein ${ }^{1}$ and Robert Scheichl ${ }^{2}$
}

\begin{abstract}
Finite element tearing and interconnecting (FETI) methods are efficient parallel domain decomposition solvers for large-scale finite element equations. In this work we investigate the robustness of FETI methods in case of highly heterogeneous (multiscale) coefficients. Our main application are magnetic field computations where both large jumps and large variation in the reluctivity coefficient may arise. We give theoretical condition number bounds which are confirmed in numerical tests.
\end{abstract}

\section{Introduction}

Finite element tearing and interconnecting (FETI) methods due to Farhat and Roux $[2,14]$ are parallel solvers for large-scale finite element (FE) systems arising from partial differential equations (PDEs). Typically, the conditioning of such FE system matrices heavily suffers from the total number of degrees of freedom (DOFs). When the number of DOFs grows large, direct solvers are out of question and efficient preconditioners for iterative solvers are required. Additionally, the parallelization of numerical algorithms gets increasingly important to date. FETI methods are known to be parallely scalable and quasi-optimal with respect to the number of DOFs. For a comprehensive presentation of FETI and related methods we refer to the monograph by Toselli and Widlund [14]. As an additional advantage, one can easily couple finite and boundary element discretizations within the same framework, resulting in

Clemens Pechstein

SFB F013 / Institute of Computational Mathematics, Johannes Kepler University, Altenberger Str. 69, 4040 Linz, Austria, e-mail: clemens . pechstein@numa . uni-linz.ac.at,

Robert Scheichl

Department of Mathematical Sciences, University of Bath, Bath BA2 7AY, United Kingdom, email: masrs@bath.ac.uk 
so-called coupled FETI/BETI methods, see $[4,6,7]$. Even exterior domains can be incorporated to model radiation conditions, see $[5,11]$

Let us briefly describe the FETI method. As a model problem we consider the finite element discretization of the Poisson-type problem

$$
-\nabla \cdot(\alpha \nabla u)=f
$$

in the bounded domain $\Omega \subset \mathbf{R}^{d}, d=2$ or 3, subject to suitable interface and boundary conditions. In Section 4 we will consider a similar equation for 2D magnetostatics. The domain $\Omega$ is partitioned into $N$ non-overlapping subdomains $\Omega_{i}$, $i=1, \ldots, N$, cf. Fig. 1, right. Introducing separate unknowns $u_{i}$ on the subdomains including the DOFs on their boundaries, we obtain the saddle point problem

$$
\left(\begin{array}{cccc}
K_{1} & & 0 & B_{1}^{\top} \\
& \ddots & & \vdots \\
0 & & K_{N} & B_{N}^{\top} \\
B_{1} & \cdots & B_{N} & 0
\end{array}\right)\left(\begin{array}{c}
u_{1} \\
\vdots \\
u_{N} \\
\lambda
\end{array}\right)=\left(\begin{array}{c}
f_{1} \\
\vdots \\
f_{N} \\
0
\end{array}\right)
$$

where $K_{i}$ are the subdomain stiffness matrices, and are $f_{i}$ the corresponding load vectors. The operators $B_{i}$ are signed Boolean matrices such that each row of the system

$$
\sum_{i=1}^{N} B_{i} u_{i}=0
$$

has the form $u_{i}\left(x^{h}\right)-u_{j}\left(x^{h}\right)=0$ for a finite element node $x^{h}$ on the interface between the subdomains $\Omega_{i}$ and $\Omega_{i}$, thus enforcing the continuity of the solution $u$. The Lagrange multiplier $\lambda$ plays the role of a continuous flux on the subdomain interfaces. Introducing a special projection $P$, the dual problem to (2) can be written in the form

$$
P F \tilde{\lambda}=\widetilde{d},
$$
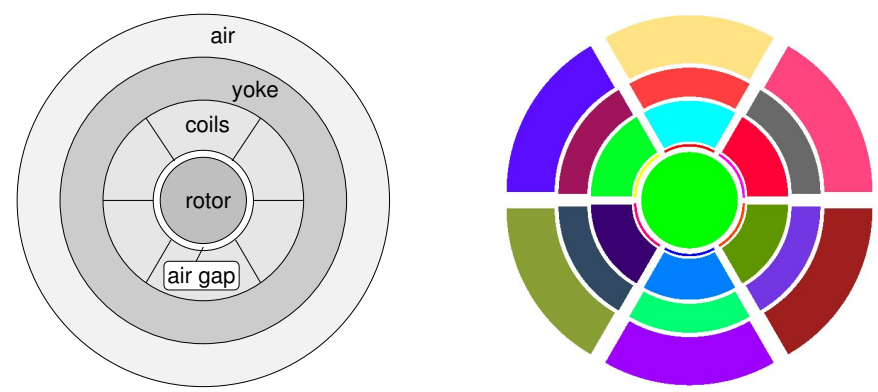

Fig. 1 Left: Model of an electric motor. Right: Possible subdomain partitioning (explosive view). 
with $F=\sum_{i=1}^{N} B_{i} K_{i}^{\dagger} B_{i}^{\top}$, where the operators $K_{i}^{\dagger}$ correspond to the solution of (possibly) regularized Neumann problems on the subdomains. The FETI method is now a special projected preconditioned conjugate gradient (PCG) method to solve problem (3). The chosen preconditioner involves the solution of local Dirichlet problems, and the projection $P$ involves the solution of a coarse problem which corresponds to a sparse linear system of dimension $\mathscr{O}(N)$. Usually, one chooses the partition in a way that the local subdomain problems can efficiently be handled by sparse direct solvers, such as $L U$-factorization with suitable pivoting. The factorizations of the local system matrices can be computed in a preprocessing phase and kept in memory during the whole FETI method. Note that these local, decoupled problems can be parallelized in a straightforward manner, e. g., treating each subdomain on a different processor. Once problem (3) is solved, the actual solution $u$ can easily be determined from the Lagrange multiplier $\widetilde{\lambda}$. The spectral condition number $\kappa$ of the preconditioned system can finally be estimated by

$$
\kappa \leq C^{*}(\alpha) \max _{i=1}^{N}\left(1+\log \left(H_{i} / h_{i}\right)\right)^{2},
$$

where the constant $C^{*}(\alpha)$ is independent of the subdomain diameters $H_{i}$, the mesh parameters $h_{i}$, and the number $N$ of subdomains. If $\alpha$ is (globally) constant, then $C^{*}(\alpha) \sim 1$. As it is well known, the number of PCG iterations needed to achieve a given accuracy, is essentially determined by $\sqrt{\kappa}$. In a parallel scheme, the total computational complexity of the FETI-PCG method is given by

$$
\mathscr{O}\left(\left(\mathscr{D}(N)+\mathscr{D}\left(N_{l o c}\right)\right) \log \left(\varepsilon^{-1}\right) \sqrt{\kappa}\right),
$$

where $N_{\text {loc }} \sim \max _{i=1}^{N}\left(H_{i} / h_{i}\right)^{d}$ is the maximal number of DOFs per subdomain, $\mathscr{D}(\cdot)$ is the cost of the direct solver, and $\varepsilon>0$ is the desired relative error reduction in the energy norm.

However, in many applications the original system matrix is ill-conditioned due to heterogeneous coefficient distributions. As we will discuss in Section 4, in magnetic field computations one may have

- large jumps in the reluctivity coefficient due to different materials, and

- smooth but large variation in the same coefficient due to nonlinear effects.

We are interested in the question whether/how the condition number $\kappa$ of the preconditioned FETI system is affected by this. If the heterogeneities are resolved by the subdomain partition (i. e., $\alpha$ constant on each $\Omega_{i}$ ), then, using a special diagonal scaling, Klawonn and Widlund [3] proved that $C^{*}(\alpha) \sim 1$. However, in general, using classical proof techniques, we only get

$$
C^{*}(\alpha) \leq C \max _{i=1, \ldots, N} \max _{x, y \in \Omega_{i}} \frac{\alpha(x)}{\alpha(y)}
$$

with $C$ independent of $\alpha$, i. e., the bound is proportional to the maximum local variation of $\alpha$ on the subdomains, which can be rather large. As noticed by several au- 
thors $[4,13]$ this asymptotic bound is in general far too pessimistic, and robustness is observed for many special kinds of coefficient distributions.

The aim of the present contribution is to give more theoretical insight on the coefficient-dependency. Due to space limitations we summarize our recent work [10] considering variation in subdomain interiors in Section 2, and we give an outlook to new theoretical results for the case of variation near the subdomain interfaces in Section 3. Finally, Section 4 deals with the application to magnetostatic problems.

\section{Variation in subdomain interiors}

In this section we give a sharper estimate than (6) for the case of variation in the subdomain interiors. On each subdomain $\Omega_{i}$ with diameter $H_{i}$ and discretization parameter $h_{i}$, we choose a width $\eta_{i} \in\left[h_{i}, H_{i} / 2\right]$ and define the boundary layer $\Omega_{i, \eta_{i}}$ by the agglomeration of those finite elements which have distance at most $\eta_{i}$ from the boundary, cf. Fig. 2, left. Under suitable assumptions on the geometric setting and the subdomain partition, we can prove the bound

$$
C^{*}(\alpha) \leq C \max _{j=1}^{N}\left(\frac{H_{j}}{\eta_{j}}\right)^{2} \max _{i=1}^{N} \max _{x, y \in \Omega_{i, \eta_{i}}} \frac{\alpha(x)}{\alpha(y)} .
$$

This bound involves only the variation of $\alpha$ in the boundary layer $\Omega_{i, \eta_{i}}$ and is independent of the variation of $\alpha$ in the subdomain interior $\Omega_{i} \backslash \Omega_{i, \eta_{i}}$. For $\eta_{j} \sim H_{j}$ we reproduce the known estimate (6), in particular our bound is still robust with respect to large jumps across the subdomain interfaces. However, if $\alpha$ exhibits large (even arbitrary) variation in the interior $\Omega_{i} \backslash \Omega_{i, \eta_{i}}$ of the subdomains, but varies little in the boundary layers, our new bound (7) is in general far better/sharper than (6). Moreover, if in addition the coefficient is larger in the interior $\Omega_{i} \backslash \Omega_{i, \eta_{i}}$ than in the boundary layer on each subdomain, then the quadratic factor $\left(H_{j} / \eta_{j}\right)^{2}$ reduces to a linear factor $H_{j} / \eta_{j}$. The detailed proof can be found in our recent paper [10].

In the following we give a two-dimensional numerical example. We partition the unit square into 25 congruent, square-shaped subdomains. The coefficient is chosen
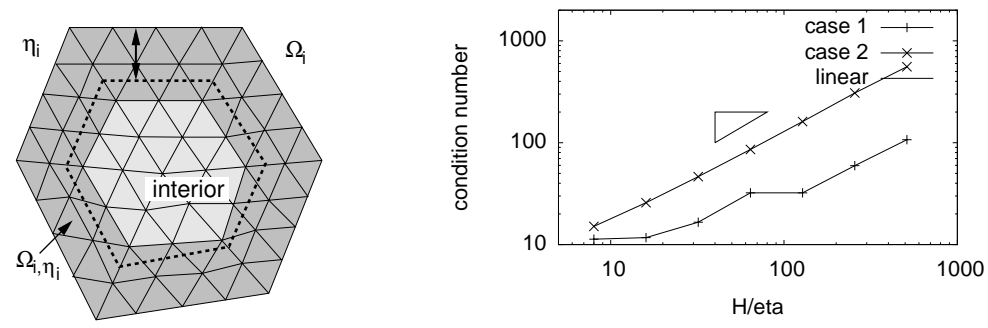

Fig. 2 Left: Subdomain boundary layer. Right: Estimated condition numbers $\kappa$ for varying width parameter $\eta$, fixed discretization parameter $h$ (logarithmic scales). 
to be $\alpha=10^{5}$ (Case 1) and $\alpha=10^{-5}$ (Case 2) in the subdomain interiors, and $\alpha=1$ on the rest. The distance between the "material" jump and the subdomain interfaces is denoted by $\eta$. We have used a globally uniform discretization with $H / h=512$. Fig. 2, right, shows the estimated condition numbers $\kappa$ of the preconditioned FETI systems for different values of the width parameter $\eta$. We see that our asymptotic bound is sharp for Case 1, but still slightly pessimistic for Case 2 .

\section{Interface variation}

In this section we would like to give an outlook on our work for interface variation which will be exposed in more detail in an upcoming paper. A key tool to the analysis of FETI methods is Poincaré's inequality,

$$
\int_{\Omega_{i}}|w(x)|^{2} d x \leq C_{P} H_{i}^{2} \int_{\Omega_{i}}|\nabla w(x)|^{2} d x,
$$

which holds for all $w \in H^{1}\left(\Omega_{i}\right)$ with vanishing mean value, i. e., $\int_{\Omega_{i}} w(x) d x=0$. The constant $C_{P}>0$ depends only on the shape of $\Omega_{i}$. A similar inequality holds if the average of $w$ over a part of the boundary $\partial \Omega_{i}$ vanishes. Concerning heterogeneous coefficients, we would be interested in an inequality of the same form but where the integrals are weighted with the coefficient $\alpha(x)$ and where the constant $C_{P}$ does not depend on $\alpha$, or at least only very mildly on the heterogeneity in $\alpha$. Such inequalities are not known in general, but we can show one for a special case.

Assume that each subdomain $\Omega_{i}$ consists of two connected subregions $\Omega_{i}^{(1)}, \Omega_{i}^{(2)}$ where $\alpha$ is mildly varying, i. e.,

$$
\underline{\alpha}_{i}^{(k)} \leq \alpha(x) \leq \bar{\alpha}_{i}^{(k)} \quad \forall x \in \Omega_{i}^{(k)}, k=1,2,
$$

with moderate ratios $\bar{\alpha}_{i}^{(k)} / \underline{\alpha}_{i}^{(k)}$; we can think of two quasi-homogeneous materials within each subdomain. Using two separate Poincaré inequalities one can show that

$$
\int_{\Omega_{i}} \alpha(x)|w(x)|^{2} d x \leq\left\{\max _{k=1,2} C_{P}^{(k)} \frac{\bar{\alpha}_{i}^{(k)}}{\underline{\alpha}_{i}^{(k)}}\right\} H_{i}^{2} \int_{\Omega_{i}} \alpha(x)|\nabla w(x)|^{2} d x,
$$

for all functions $w \in H^{1}\left(\Omega_{i}\right)$ which have vanishing mean value over a connected part $\Lambda_{i}$ of the interface $\partial \Omega_{i}^{(1)} \cap \partial \Omega_{i}^{(2)}$, i. e., $\int_{\Lambda_{i}} w(x) d s_{x}=0$. The constants $C_{P}^{(1)}$ and $C_{P}^{(2)}$ depend only on the shapes of the subregions $\Omega_{i}^{(1)}$ and $\Omega_{i}^{(2)}$ respectively, and on the relative shape of $\Lambda_{i}$. For a variant of FETI called all-floating FETI method $[1,8,9]$, our Poincaré type inequality (8) eventually allows a proof of the bound

$$
C^{*}(\alpha) \leq C \max _{i=1}^{N} \max _{k=1,2} \frac{\bar{\alpha}_{i}^{(k)}}{\underline{\alpha}_{i}^{(k)}},
$$



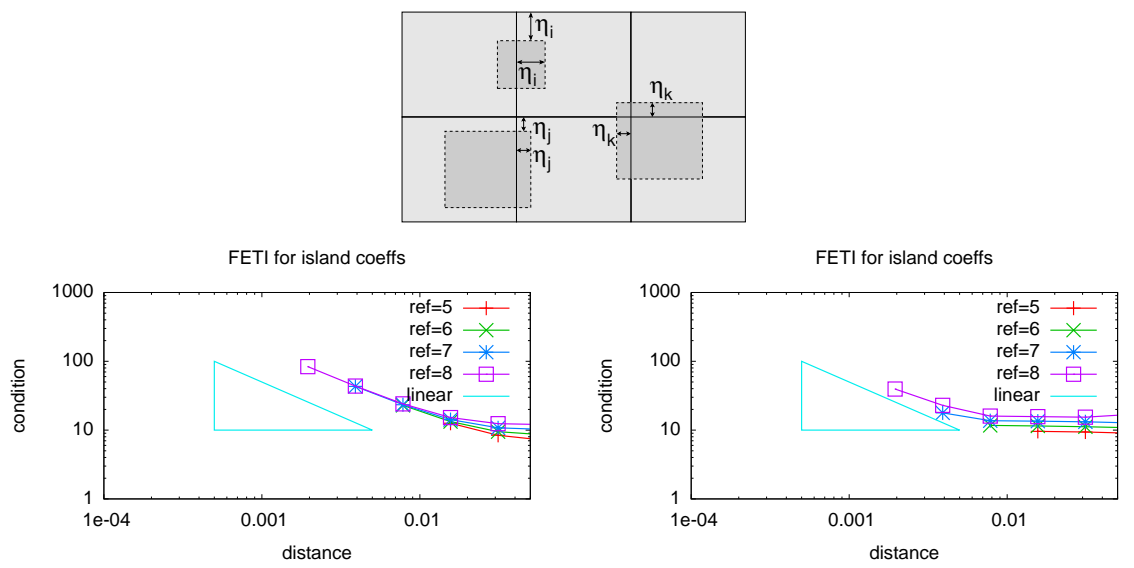

Fig. 3 Upper: Sketch of coefficient "islands" cutting through edges and crosspoints of the subdomain partitioning. Lower left: Condition numbers for "edge islands". Lower right: Condition numbers for "crosspoint islands".

where the constant $C$ is independent of $H_{i}, h_{i}, N$, and $\alpha$, but it depends on the geometry of the subregions $\Omega_{i}^{(k)}$. Combining this idea with the theory from Section 2, one can even allow three qualitatively different subregions per subdomain:

- two connected subregions of mild variation in $\alpha$ that cover the boundary layer $\Omega_{i, \eta_{i}}$ of the subdomain, and

- a remaining part contained in the subdomain interior, where arbitrary variation of $\alpha$ can be allowed.

Under suitable assumptions on the shapes of these subregions it is again possible to give explicit bounds for $C^{*}(\alpha)$ involving (9) and the ratios $H_{i} / \eta_{i}$ similar to (7). For numerical examples we have tested so-called coefficient "islands" which cut through an $e d g e$, i. e., the interface of two subdomains, or which contain a crosspoint of four subdomains, cf. Fig. 3, upper. A suitable choice for $\eta$, the witdh of the boundary layer, is also indicated in that figure. Note, however, that we have only tested one island at a time. In each example we have set the coefficient $\alpha=10^{5}$ in the island, and $\alpha=1$ elsewhere. The estimated condition numbers for different values of $\eta$ and different levels of mesh refinement are depicted in Fig. 3, lower.

\section{Application to magnetostatic problems}

In the case of nonlinear magnetostatics in two dimensions (transverse magnetic mode), we have to solve

$$
-\nabla \cdot\left[v_{i}(|\nabla u|) \nabla u\right]=f \quad \text { in } \Omega_{i},
$$


subject to suitable interface and boundary conditions, where $u$ is the $z$-component of the magnetostatic vector potential, and $v_{i}$ is the reluctivity. For linear materials, $v_{i}$ is constant. For other materials, such as ferromagnetic ones, the reluctivity $v_{i}$ depends nonlinearly on the magnetic flux density $|\mathbf{B}|=|\nabla u|$, and it is defined by the material law $\mathbf{H}=v_{i}(|\mathbf{B}|) \mathbf{B}$ in $\Omega_{i}$, where $\mathbf{H}$ denotes the magnetizing force (note that we restrict ourselves to isotropic materials and neglect hysteresis). In our numerical computations we use realistic approximations of such material curves using the interproximation method proposed in [12]. If we apply Newton's method to (10), the linearized system in each Newton step is of similar form as problem (1), only that we obtain a matrix-valued coefficient which depends on the current Newton iterate $u^{(k)}$, see, e. g., [4]. For many material curves, the variation of the coefficient depends mainly on the variation of $|\mathbf{B}|$. However, the flux density $|\mathbf{B}|$ may vary strongly along subdomain boundaries and large values of $|\mathbf{B}|$ appear mostly at singularities of the potential $u$, e.g., near material corners.

Contrary to the usual suggestion to choose subdomain partitions that resolve material interfaces in order to obtain robustness (for numerical examples see $[4,5]$ ), our new bounds (7), (9) suggest that it might be more advantageous to put each peak of $|\mathbf{B}|$ and thus each material corner into the center of a subdomain. Fig. 4 shows two such examples. In both cases, the coefficient variation is approximately $7 \times 10^{3}$ but our FETI solver performs extremely well (Case 1: condition number 8.5, Case 2: condition number 13.7, compared to 8.3 for a globally constant coefficient). Our theory for interior variation (Section 2) can perfectly explain the low condition number in Case 1 since the boundary variation is small. Inspecting Case 2, we find that there are indeed two regions contained in the boundary layer with qualitatively different coefficients, see the jump in Fig. 4, lower right. Thus, Section 3 partially explains why the condition number is still quite robust with respect to the highly heterogeneous coefficient.

Acknowledgements We would like to thank Ulrich Langer for his encouragement. The first author acknowledges the financial support by the Austrian Science Funds (FWF), grant SFB F013.

\section{References}

1. Zdeněk Dostál, David Horák, and Radek Kučera. Total FETI - An easier implementable variant of the FETI method for numerical solution of elliptic PDE. Commun. Numer. Methods Eng., 22(12):1155-1162, 2006.

2. C. Farhat and F.-X. Roux. A method of finite element tearing and interconnecting and its parallel solution algorithm. Int. J. Numer. Meth. Engrg., 32(6):1205-1227, 1991.

3. A. Klawonn and O. B. Widlund. FETI and Neumann-Neumann iterative substructuring methods: Connections and new results. Comm. Pure Appl. Math., 54(1):57-90, 2001.

4. U. Langer and C. Pechstein. Coupled finite and boundary element tearing and interconnecting solvers for nonlinear potential problems. ZAMM Z. Angew. Math. Mech., 86(12), 2006.

5. U. Langer and C. Pechstein. Coupled FETI/BETI solvers for nonlinear potential problems in (un)bounded domains. In G. Ciuprina and D. Ioan, editors, Scientific Computing in Electrical 

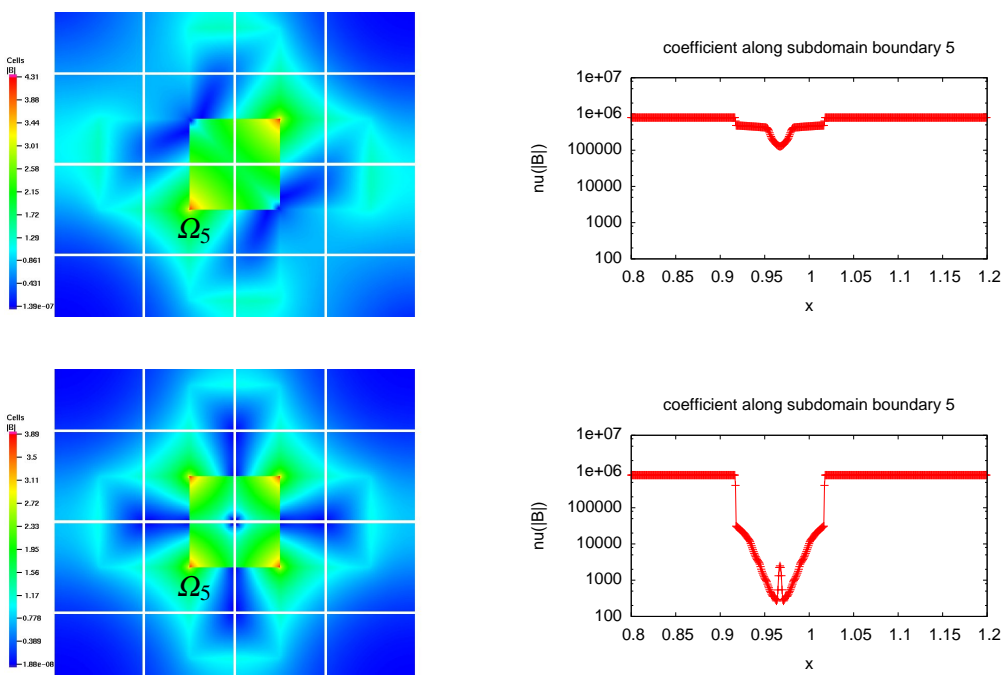

Fig. 4 Upper: Case 1. Lower: Case 2. Left: $|\mathbf{B}|$-field, subdomain partition. Right: Coefficient $v(|\mathbf{B}|)$ plotted along the boundary of subdomain $\Omega_{5}$.

Engineering, volume 11 of Mathematics in Industry: The European Consortium for Mathematics in Industry. Springer, Berlin, Heidelberg, 2007.

6. Ulrich Langer and Olaf Steinbach. Boundary element tearing and interconnecting method. Computing, 71(3):205-228, 2003.

7. Ulrich Langer and Olaf Steinbach. Coupled boundary and finite element tearing and interconnecting methods. In Lecture Notes in Computational Sciences and Engineering, volume 40, pages 83-97. Springer, Heidelberg, 2004.

8. Günther Of. BETI-Gebietszerlegungsmethoden mit schnellen Randelementverfahren und Anwendungen. PhD thesis, Universität Stuttgart, Germany, January 2006.

9. Günther Of. The all-floating BETI method: Numerical results. In Domain Decomposition Methods in Science and Engineering XVII, volume 60 of Lecture Notes in Computational Science and Engineering, pages 295-302. Springer, Berlin, Heidelberg, 2008.

10. C. Pechstein and R. Scheichl. Analysis of FETI methods for multiscale PDEs. To appear in Numerische Mathematik, 2008; for a preprint see http://www.ricam. oeaw.ac.at/ publications/reports/08/rep08-20.pdf.

11. Clemens Pechstein. Boundary element tearing and interconnecting methods in unbounded domains. To appear in Applied Numerical Mathematics, 2008.

12. Clemens Pechstein and Bert Jüttler. Monotonicity-preserving interproximation of $B$ - $H$-curves. Journal of Computational and Applied Mathematics, 196(1):45-57, 2006.

13. D. Rixen and C. Farhat. A simple and efficient extension of a class of substructure based preconditioners to heterogeneous structural mechanics problems. Internat. J. Numer. Methods Engrg., 44(4):489-516, 1999.

14. A. Toselli and O. B. Widlund. Domain Decoposition Methods - Algorithms and Theory, volume 34 of Springer Series in Computational Mathematics. Springer, Berlin, Heidelberg, 2005. 